Proc. Indian Acad. Sci. (Chem. Sci.), Vol. 105, No. 6, December 1993, pp. 463-474.

(C) Printed in India.

\title{
Kinetics of charge transfer reactions in photoelectrochemical cells
}

\author{
R MEMMING \\ FB Physik der Carl-von-Ossietzki-Universität Oldenburg and Institut für Solarenergiefor- \\ schung (ISFH), Sokelantstraße 5, D-3000 Hannover 1, Germany

\begin{abstract}
In the present paper, fundamental aspects of charge transfer processes at semiconductor particles and extended electrodes are analyzed and compared. Although, in principle, the same reactions occur at particles and electrodes, different factors, such as light intensity, particle size, adsorption of electron donors or acceptors and formation of space charges, influence the reaction rates and sometimes even the reaction route. It is shown that rate constants are preferably determined from measurements at extended electrodes. Various mechanisms are discussed in detail.
\end{abstract}

Keywords. Semiconductor-electrodes; semiconductor-particles; kinetics.

\section{Introduction}

For almost 20 years many research groups have been studying and developing solid state devices, photoelectrochemical and photochemical systems with respect to conversion of solar energy. From this research it has became quite clear that high conversion efficiencies can only be obtained with heterogeneous systems. From this point of view, systems containing solid-solid and solid-liquid interfaces are of particular interest. Solid-solid systems, such as for instance $p n$-junctions, are adequate for conversion of solar energy into electricity, whereas semiconductor-liquid systems can, in principle, be used for the production of electricity as well as of storable chemical fuel.

During the last decade, many reactions in semiconductor suspensions and colloidal solutions besides processes at extended electrodes have been extensively studied. Various aspects of this research have been summarized in review articles. Restricting ourselves to the last decade, review articles have been published on photoelectrochemical conversion by Gerischer (1979), Tributsch $(1982,1988)$, Lewis (1984), Hodes (1985), Memming $(1988,1990,1991)$, on charge transfer reactions at semiconductor electrodes by Pleskov and Gurevick (1986), Morrison (1980), Jaegermann and Tributsch (1988), Memming (1983, 1993) and Gomes and Cardon (1982) and on semiconductor particles by Henglein $(1988,1989)$ and Bahnemann (1991). In the present paper, the kinetics of reactions at semiconductor electrodes and particles will be compared with the main emphasis on the question of which factors determine the reaction rates in both cases.

\section{Reactions at semiconductor particles}

Since a semiconductor crystal or particle is a multi-atom or a multi-electron system, the electron energy levels are usually degenerated into energy bands. The distance 
between the conduction and valence bands can be determined by absorption measurements, from which an absorption edge can be determined. The energy position of the conduction and valence band is usually entirely determined by the liquid contacting the semiconductor electrode or particle. This result is due to the strong interaction between the semiconductor surface and the electrolyte, such as for instance water, as proved experimentally by capacity measurements, which can be performed only with electrodes and not with particles (see also §3). Accordingly, the positions of the conduction and valence bands at the surface of a semiconductor do not change upon addition of an electron donor or acceptor, such as a redox system, to the electrolyte. The absorption of the colloidal particles changes, however, if their dimensions become smaller than about $5 \mathrm{~nm}$. On decreasing the size, at first a larger gap is observed, the finally - at very small diameter - exciton bands occur in the absorption spectra. Since the long range interaction disappears, quantization of the energy bands occurs. The exciton formation in small particles is due to a stronger coulomb interaction within the limited space in small particles. The energy levels in a particle have been calculated by using a simple quantum mechanical model for a particle in a 3-dimensional box (see e.g. Weller et al (1984), Rosetti et al (1985)). An exact position of the energy levels of the extremely small particles cannot be determined. One can only estimate it by studying electron transfer reactions from the conduction band of the particle to an electron acceptor during light excitation. One example is the reduction of protons at $5 \mathrm{~nm}$ PbSe- and $\mathrm{HgSe}$-colloids, which has only been observed with small and not with big particles (Nedeljkovic et al 1986; Micic et al 1992). Accordingly, the conduction band of big particles occurs at energies below the $\mathrm{H}^{+} / \mathrm{H}_{2}$-redox potential and above it in the case of quantization.

Concerning photo-induced charge-transfer reactions at small or big semiconductor particles, such processes are only possible if both oxidation by hole transfer and reduction by electron transfer occur simultaneously, as illustrated in figure $1 \mathrm{~b}$. Accordingly, the slowest reaction step determines the overall rate, as expected in the case of excited aromatic molecules also. On the other hand, if no electron acceptor
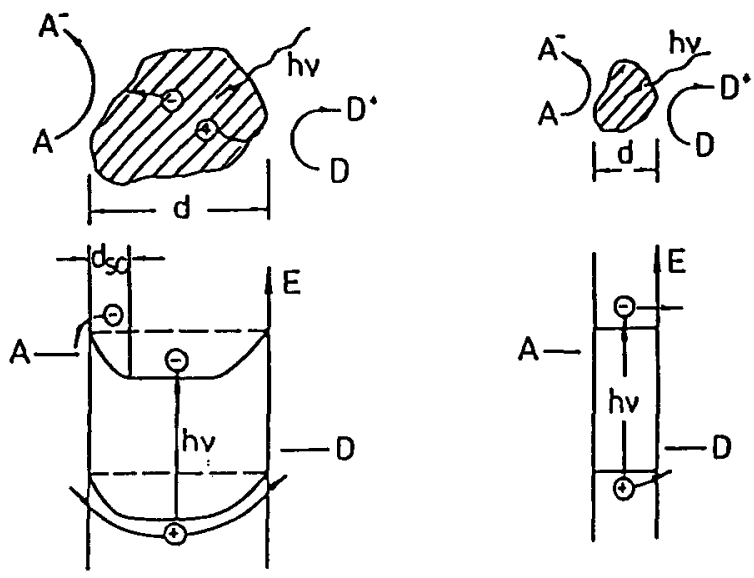

o) $d>d_{s c}$

b) $d \ll d_{S c}$

Figure 1. Electron- and hole transfer at large (a) and small (b) semiconductor particles to an electron acceptor $\mathrm{A}$ and donor $\mathrm{D}$. 
is present in the solution, then the photoexcited electrons may be trapped at surface sites, as found with colloidal $\mathrm{TiO}_{2}$ (Bahnemann et al 1984). These electrons exhibit a characteristic transient absorption spectrum, which can be easily measured by laser flash spectroscopy because of the large surface area of the $\mathrm{TiO}_{2}$ colloidal solution. Also the trapping of holes has been observed when electrons are efficiently scavenged. Corresponding transient spectra of trapped holes have been found with $\mathrm{TiO}_{2}$ (Bahnemann et al 1984) and CdS-particles (Baral et al 1986).

As discussed above, electrons and holes produced by light excitation in a small semiconductor particle can be easily transferred to an electron-acceptor and -donor, respectively, provided that the energetic requirements are fulfilled. The quantum efficiency of the reaction depends on the transfer rate at the interface, on the recombination rate within the particle and on the transit time, the latter being given by (Grätzel and Frank 1982),

$$
\tau=R^{2} / \pi^{2} D
$$

where $R$ is the radius of the particle and $D$ the diffusion coefficient of the excited charge carriers. Taking a typical value of $D \approx 0.1 \mathrm{~cm}^{2} \mathrm{~s}^{-1}$ and a radius of $10 \mathrm{~nm}$, the average transit time is only about $1 \mathrm{ps}$. This value is much smaller than that for recombination, which is usually greater than several nanoseconds. Accordingly, the kinetics of a reaction is mainly determined by the surface properties of a particle. Besides surface effects, particles behave like excited organic molecules.

Mostly, oxidation and reduction of organic molecules have been studied in semiconductor suspensions and colloidal solutions. This implies that two charges per molecule have to be transferred before a stable state is reached. Taking the oxidation of ethanol as an example, the transfer of two electrons to a particle leads to the formation of aldehyde. This reaction has been studied at different semiconductor particles such as $\mathrm{TiO}_{2}, \mathrm{CdS}$ and $\mathrm{ZnS}$ by using $\mathrm{H}_{2} \mathrm{O}$ or $\mathrm{O}_{2}$ as an electron acceptor. In the case of $\mathrm{TiO}_{2}, \mathrm{SnO}_{2}$ and $\mathrm{CdS}$, only acetaldehyde is formed, whereas with small $\mathrm{ZnS}$-particles butanediol was also formed as an oxidation product (Müller et al 1993). The latter result can be explained by a single-hole transfer from an excited ZnS-particle to ethanol, leading to the formation of a hydroxyethyl radical which diffuses into the solution. There it undergoes dimerization and disproportionation by reacting with another radical, as illustrated in figure 2 (Müller et al 1993). A similar mechanism has been proposed by Henglein et al (1984), who observed the formation of pinalcol at $\mathrm{nm}$-particles of $\mathrm{ZnS}$ in the presence of 2-propanol, using $\mathrm{CO}_{2}$ as an electron acceptor. Cross-reaction products have also been reported. For instance, Yanagida (1991 private communication) has observed the formation of pinalcol at small CdS-colloids with an excess of $10 \mathrm{~mol} \%$ of $\mathrm{S}^{2-}$ when using methanol as an electron donor and benzophenone as an acceptor. It should be mentioned that some published experimental data on reaction products at $\mathrm{ZnS}$ - and CdS-colloids are controversial. This is due to very complex surface chemistry, as studied in detail by Müller et al (1993). Already the synthesis of the particles plays an important role, for instance, whether the ZnSparticles are prepared in a solution with an excess of $\mathrm{S}^{2-}$ - or $\mathrm{Zn}^{2+}$-ions. A discussion of the influence of the surface chemistry on the kinetics, however, would be beyond the scope of the present paper.

It is interesting to note that acetaldehyde but no butanediol is formed at excited $\mathrm{TiO}_{2}, \mathrm{SnO}_{2}$ and CdS-particles. Here also a hole is transferred to ethanol, leading to 

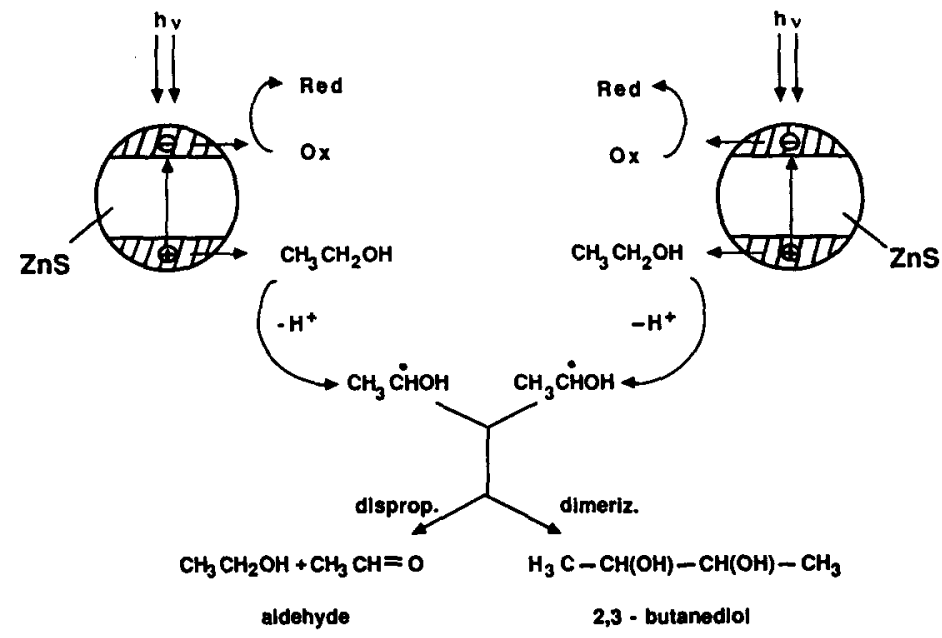

Figure 3. Two-step oxidation of ethanol at small $\mathrm{TiO}_{2}$-particles ("current doubling effect") during illumination.

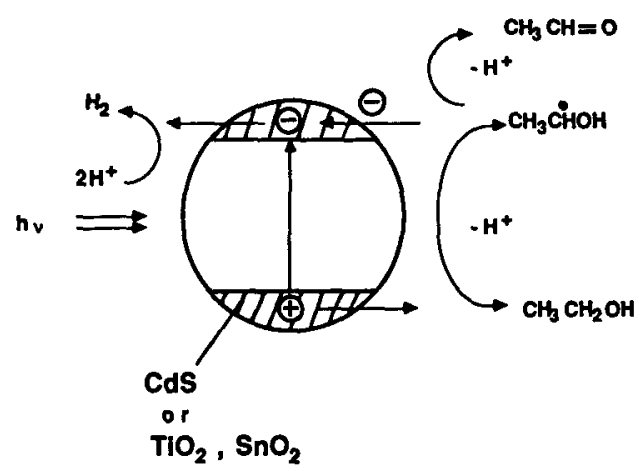

Figure 3. Two-step oxidation of ethanol at small $\mathrm{TiO}_{2}$-particles ("current doubling effect") during illumination.

the formation of a radical. Since, however, the energy bands occur at much lower energies, an electron can be injected into the conduction band of these materials (figure 3), i.e. aldehyde is formed immediately at the same particle. Here, a second electron is created in the conduction band besides the other one produced by light excitation. This is possible because in a semiconductor-particle the density of energy states is high. In this aspect, even a small particle differs considerably from an excited aromatic molecule. The different behaviour of $\mathrm{ZnS}$ as compared to the other semiconductors becomes clear by comparing the position of the energy bands with respect to the redox potentials of the ethanol/radical- and of the radical/aldehyde-couples, the latter being determined by Lilie et al (1971) (see figure 4). The phenomenon that both energy bands are involved in the oxidation of organic molecules is well known from investigations with semiconductor electrodes ("current-doubling-effect"). For details see e.g. Memming (1983). 


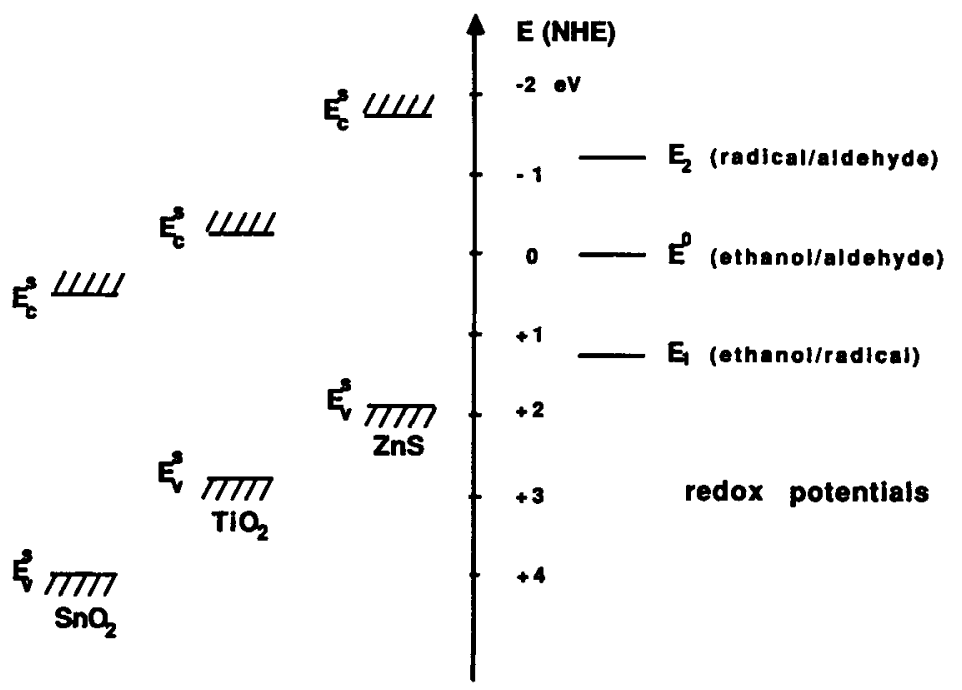

Figure 4. Energy scheme of various semiconductors (left) and redox potentials of the ethanol/aldehyde system (right).

Returning to the oxidation of $\mathrm{C}_{2} \mathrm{H}_{5} \mathrm{OH}$ to the radical by a single hole transfer at an excited $\mathrm{ZnS}$-particle, the question arises as to why this radical is not immediately further oxidized to the aldehyde by a second hole transfer, which is energetically feasible. Concerning this problem, it has to be realized that this second hole has also to be generated by light excitation, and that it may take a considerable time until a second photon is absorbed by the same particle. This time interval can be estimated as follows.

Taking a 3-nm ZnS-particle as an example, its absorption cross-section $\sigma$ can be calculated from the Mie-theory (Ribarsky 1985). We thus obtained $\sigma=5.4 \times 10^{-16} \mathrm{~cm}^{-2}$ at a wavelength of $320 \mathrm{~nm}$. Using light intensity of $3 \times 10^{16}$ photons $\mathrm{cm}^{-2} \mathrm{~s}^{-1}$, the time interval between two absorption incidents is, on the average, about $60 \mathrm{~ms}$. Within this period, a radical formed by hole transfer can certainly diffuse away from the particle, which explains the formation of butanediol in the solution (figure 2). Since the absorption cross-section increases with the diameter of the particle, the time interval between two absorption incidents in an individual particle decreases. For instance, in the case of a 4- $\mu \mathrm{m}$-particle $\sigma=1.3 \times 10^{-7} \mathrm{~cm}^{-2}$, and the time interval is of the order of $100 \mathrm{ps}$ for the same photon flux, i.e. it is shorter than for 3-nm-particles by 8 orders of magnitude. According to this estimate, it was predicted several years ago that the reaction mechanism may change due to this particle size effect (Memming 1988). Recently, we succeeded in proving this effect also by studying the oxidation of ethanol at small ( $3 \mathrm{~nm})$ and big $(4 \mu \mathrm{m}) \mathrm{ZnS}$-particles. As shown in figure 5, butanediol is only formed at the $3 \mathrm{~nm}$-particles, whereas aldehyde was formed with $4 \mu \mathrm{m}$-particles (Müller et al 1993).

Electrochemical investigations with semiconductor electrodes have shown that a space charge layer exists below the surface, i.e. energy bands are bent. This occurs of course also in a particle, as already illustrated in figure 1a. The thickness of the space charge layer depends on the doping, i.e. it is large for a low doping density and small for a large doping. Typical values of the thickness are of the order of 


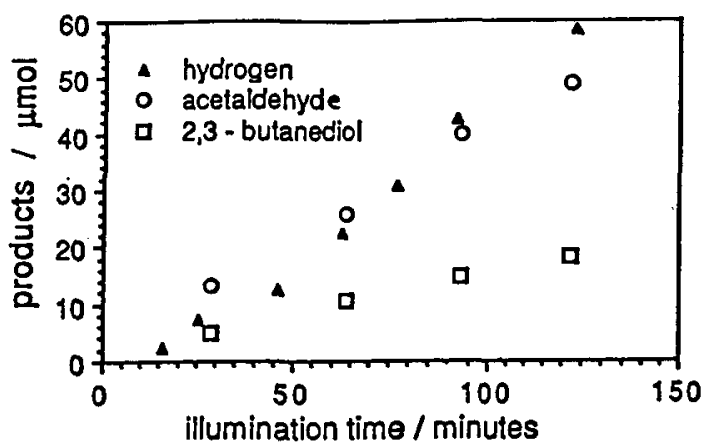

a)

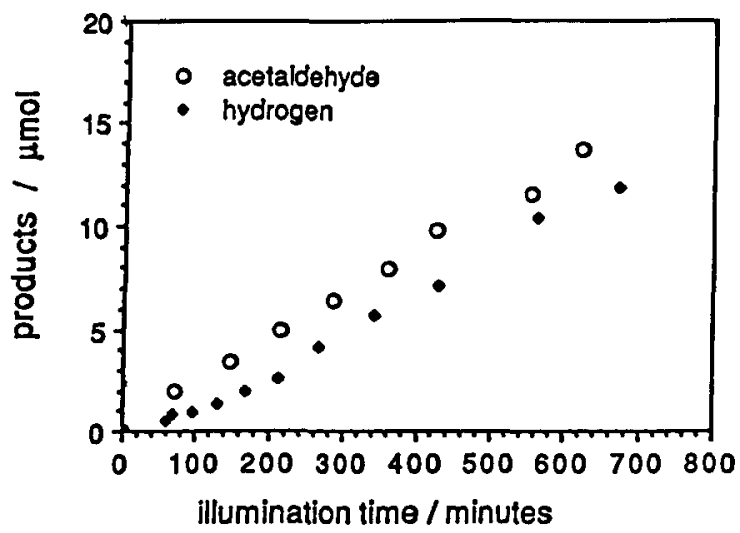

b)

Figure 5. Formation of products upon illumination of $\mathrm{ZnS}$-particles (17 $\mathrm{mol}_{\%} \% \mathrm{SH}^{-}{ }^{-}$-excess) in EtOH/ $\mathrm{H}_{2} \mathrm{O}$ (ratio 1:5) at $p \mathrm{H} \mathrm{10:} \mathrm{(a)} 3 \mathrm{~nm} \mathrm{ZnS-particles;} \mathrm{(b)} 4 \mu \mathrm{m}$ ZnS-particles. From Müller et al (1993).

$d_{\mathrm{sc}} \approx 10^{-5} \mathrm{~cm}$ for a density of $10^{17} \mathrm{~cm}^{-3}$. Since there is an energy barrier for electrons (figure 1a), the question arises whether this barrier influences the kinetics and therefore the transfer rate of reactions at particles of a size $d \gg d_{s c}$. Since the electron density at the surface can be rather small, the rate of the electron transfer to an acceptor and consequently the rate of the overall reaction will be small. In such a case, the recombination rate will increase. On the other hand, the holes created by light excitation are driven towards the surface because of the electrical field across the space charge layer. This leads to a negative charging of the particle, causing a flattening of bands, so that electrons are more easily transferred (see dashed line in figure 1a). The actual rates of the two processes, i.e. electron and hole transfer, finally depend on the rate constants.

\section{Reactions at extended electrodes}

Frequently it is difficult to get an insight into the mechanism of reactions at particles because the two processes, reduction of the electron-acceptor and oxidation of the donor, always take place at the same time. In many cases, it is easier to study reactions at semiconductor electrodes because one of two processes can be avoided by polarizing 


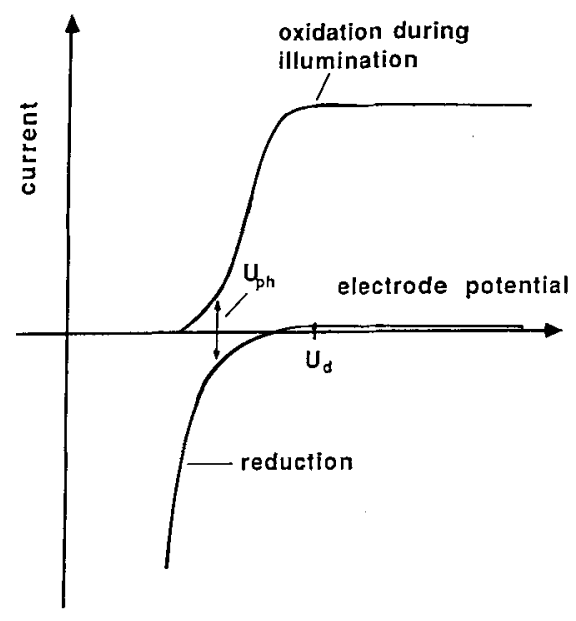

Figure 6. Schematic current-voltage characteristic for an $n$-type semiconductor electrode.

the electrode. The charge transfer process can be analyzed in terms of partial currents in the dark and under illumination, as schematically illustrated for an $n$-type semiconductor electrode in figure 6 . The cathodic current corresponds to the reduction of an electron acceptor and the anodic current to the oxidation of a donor. Since the latter current is increased by illumination, the corresponding process requires holes. Under open circuit conditions $(j=0)$, the electrode potential occurs at $U_{d}$, under illumination the open circuit potential is shifted to $U_{p h}$, i.e. to a potential at which anodic photocurrent and cathodic dark current are equal. This shift to the open circuit potential is the same as that already discussed for the big particles in $\S 2$ (see also figure 1a) and means a decrease of band bending. Assuming again that the cathodic reaction is a conduction band process, the current is given by

$$
j_{c}^{-}=e k_{c, r e d} c_{o x} n_{s},
$$

in which $c_{o x}$ is the concentration of the electron acceptor in a number of molecules or ions per cubic centimeter, $n_{s}$ the electron density $\left(\mathrm{cm}^{-3}\right)$ and $k_{c, \text { red }}$ a second-order rate constant (in $\mathrm{cm}^{4} \mathrm{~s}^{-1}$ ). Using the theories of Marcus (1964) and of Gerischer $(1960,1961)$, the rate constant is given by

$$
k_{c, \text { red }}=k_{c, \text { red }}^{\max } \exp \left[-\left(E_{c}-E_{F, \text { redox }}^{0}+\lambda\right)^{2} / 4 k T \lambda\right]
$$

in which $E_{c}$ is the lower edge of the conduction band, $E_{F, \text { redox }}$ the standard energy potential of the redox system (electron acceptor) and $\lambda$ the reorientation energy. $k_{c, \text { red }}^{\max }$ is the maximum rate constant for $\left(E_{c}-E_{F, \text { redox }}^{0}+\lambda\right)=0$. The electron density at the surface $n_{s}$ is related to the bulk density $n_{0}$ by

$$
n_{s}=n_{0} \exp \left(-e \phi_{s c} / k T\right),
$$

in which $\phi_{s c}$ is the potential across the space charge layer, as shown in figure $7 \mathrm{~b}$. Inserting (4) into (2), one has:

$$
j^{-}=e k_{c, r e d} c_{o x} n_{0} \exp \left(-e \phi_{s c} / k T\right) .
$$



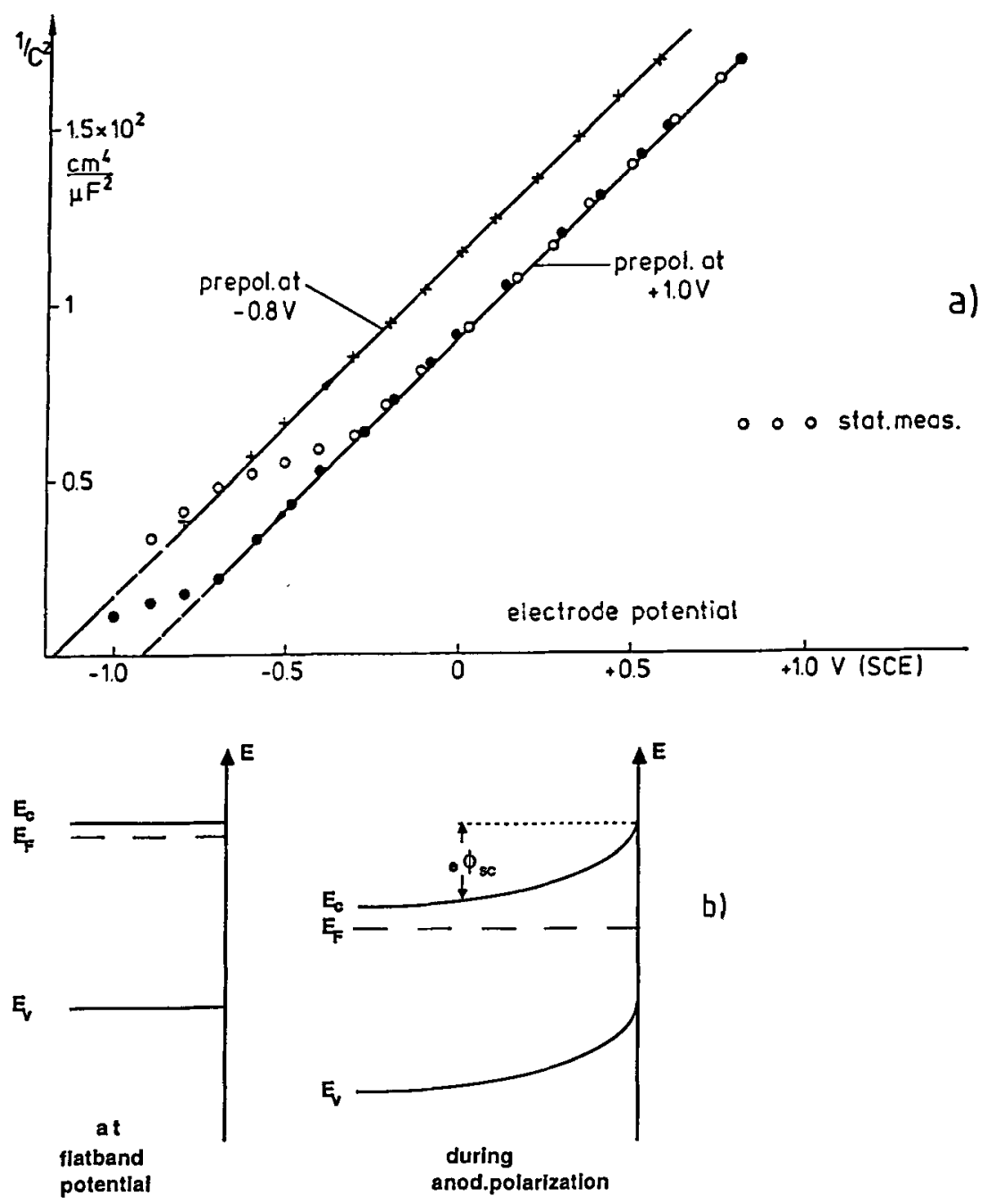

Figure 7. (a) Mott-Schottky plot of the space charge capacity of $n-\mathrm{GaAs}$ in $0 \cdot 1 \mathrm{M} \mathrm{H}_{2} \mathrm{SO}_{4}$ after anodic $(+1 \mathrm{~V})$ and cathodic $(-0.8 \mathrm{~V})$ prepolarization. (b) Energy bands at flatband potential and at an anodic potential. From Schröder and Memming (1985).

According, the cathodic dark current increases exponentially with the potential across the space-charge layer. The latter can be determined by measurements of the spacecharge capacity, which is given by the Mott-Schottky equation (Memming 1983),

$$
1 / C_{s c}^{2}=\left(\frac{2 L_{D, \text { eff }}}{\varepsilon \varepsilon_{0}}\right)^{2}\left(\frac{e \phi_{s c}}{k T}-1\right),
$$

in which $\varepsilon$ is the dielectric constant of the semiconductor, $\varepsilon_{0}$ the permittivity of the free space and $L_{D \text {, eff }}$ the effective Debye length given by

$$
L_{D, \text { eff }}=\left[\left(\varepsilon \varepsilon_{0} k T / 2 n_{0} e^{2}\right)\right]^{1 / 2} .
$$

Corresponding measurements have been performed with many semiconductor electrodes, one example ( $n$-GaAs) is given in figure $7 \mathrm{a}$. Since $1 / C_{s c}^{2}$ is plotted versus the electrode 
potential $U_{E}$, an extrapolation of the curve to $1 / C_{s c}^{2} \Rightarrow 0$ yields the so-called flatband potential $\left(U_{E}=U_{f b}\right)$. Accordingly we have

$$
U_{s c}=U_{E}-U_{f b}
$$

Using this question, one should predict from (5) that a plot of $\log j^{-}$vs. $\phi_{s c}$ or $U_{E}$ should yield a straight line with a slope of $60 \mathrm{mV} /$ decade. The rate constant can be obtained by extrapolating such a curve to the equilibrium or redox potential and determining it by using (5). The potential across the space charge layer $\phi_{s c}^{0}$ for the equilibrium case can also be taken from the capacity measurement.

Unfortunately, not many current-potential curves have been evaluated according to this model. There is one example $(n-\mathrm{ZnO})$ given in the literature where the cathodic current really increases at $60 \mathrm{mV} /$ decade, as required according to (5). Morrison (1969) has investigated the current-potential behavior of $\mathrm{ZnO}$ in aqueous electrolytes using various redox systems (electron acceptors). Evaluating these results in terms of rate constants, one obtains values of $k_{c, r e d} \leqslant 10^{-18} \mathrm{~cm}^{4} \mathrm{~s}^{-1}$, depending on the electron acceptor (Memming 1993). These data are in accordance with theoretical values obtained by using Marcuś (1964) or Gerischer's (1970) theories. Recently, Lewis (1991) has estimated from these theories a maximum rate constant of $k_{c, \text { red }}^{\max }=10^{-17}$ to $10^{-16} \mathrm{~cm}^{4} \mathrm{~s}^{-1}$ (compare also with (3)). Rate constants of $k_{c, \text { red }} \leqslant 10^{-18} \mathrm{~cm}^{4} \mathrm{~s}^{-1}$ determined experimentally are reasonable if one inserts values of the reorientation energy of $\lambda=0.5$ to $1.0 \mathrm{eV}$ into (3).

However, there are several other current-potential curves published in the literature where these curves exhibit a slope considerably larger than $60 \mathrm{mV} /$ decade. The origin is not clear yet. In some cases it may be due to a change of the potential across the Helmholtz layer because of a change of the surface coverage. For instance, the Mott-Schottky curve may be different for anodic and cathodic prepolarization, as shown for $n$-GaAS in figure 7a. Obviously, here a hydroxyl surface being formed during anodic prepolarization, is changed into a hydride surface after cathodic prepolarization (Schröder and Memming 1985). This leads to a shift of the energy bands at the surface. Since such a shift also changes the energy distance between conduction band $\left(E_{c}\right)$ and the standard redox potential $\left(E_{F, \text { redox }}^{0}\right)$ in the exponent in (3), the rate constant is also changed. Recently, the kinetics of hydrogen evolution at $n$-GaAs electrodes has been studied in more detail by performing impedance spectroscopic and current-potential measurements simultaneously. However, protons act here as electron acceptors $(p \mathrm{H} 3.5)$. From impedance spectroscopy analysis the space charge capacity $C_{s c}$ was determined (Uhlendorf 1993). Since there is a fixed relation between $C_{s c}$ and $\phi_{s c}(6)$, the potential across the space charge capacity could be determined for all currents. The result, i.e. $j^{-}$vs. $\phi_{s c}$, is given by the solid line in figure 8. Here, the slope of $\log j^{-}$vs. $\phi_{s c}$ corresponds to $60 \mathrm{mV} / \mathrm{decade}$, as required by (5). This result is of special interest because the rate constant derived from this curve is of the order of $k_{c, \text { red }}=10^{-12} \mathrm{~cm}^{4} \mathrm{~s}^{-1}$, i.e. it is 4 to 5 orders of magnitude higher than the maximum value of $k_{c, \text { red }}^{\max }=10^{-16}$ to $10^{-17} \mathrm{~cm}^{4} \mathrm{~s}^{-1}$ estimated theoretically. On the other hand, an excellent fit can be obtained by comparing the experimental curve with a theoretical $j_{c}^{-}-\phi_{s c}$-curve derived from the thermionic emission model, the latter being given by Memming (1987):

$$
j_{c}^{-}=A T^{2}\left(\frac{m^{*}}{m_{e}}\right)^{-1 / 2} \frac{n_{0}}{N_{c}} \exp \left(-\frac{e \phi_{s c}}{k T}\right),
$$




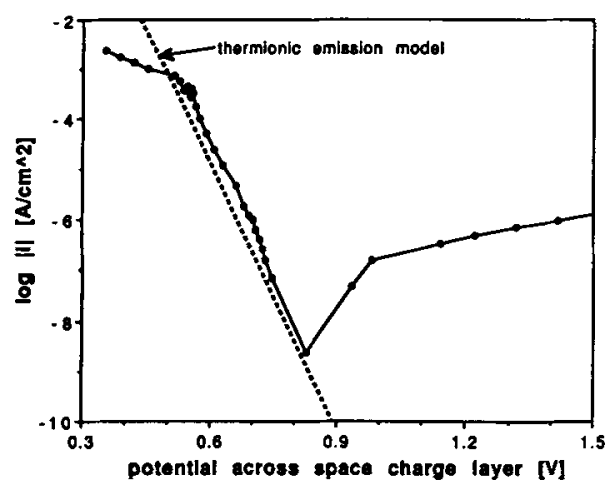

Figure 8. Current vs. potential across the space charge layer at $n$-GaAs in $\mathrm{H}_{2} \mathrm{SO}_{4}(p \mathrm{H} 3$ ); solid line - experimental data; dashed line - theoretical curve calculated from the thermionic emission model. (From Uhlendorf et al 1993).

in which $m^{*}$ is the effective electron mass and $m_{e}$ that in free space, $N_{c}$ the density of energy states at the lower edge of the conduction band, and $A$ is a constant $=120 \mathrm{~A}$ $\mathrm{cm}^{-2} \mathrm{~K}^{-2}$. This equation has been derived originally for semiconductor-metal junctions on the basis that all electrons reaching the surface are transferred with a probability of unity, i.e. the current is actually determined by the electron transport from the bulk to the surface of the semiconductor (see e.g. Sze 1981).

The question arises as to how to explain such a high rate constant. In connection with this problem it should be mentioned that the analysis of the impedance spectroscopy measurements is based on a model in which hydrogen atoms are bonded or adsorbed on the electrode surface (Butler-Vollmer or Heyrovsky mechanism), which cannot be derived here. Accordingly, an electron transfer between the semiconductor and an adsorbed electron acceptor is involved, and obviously this is extremely fast, so that the electron transport in the semiconductor is a limiting step. There are a few other current-potential curves published in the literature which also indicate an extremely high rate constant. Examples are the reduction of $\mathrm{H}_{2} \mathrm{O}_{2}$ at $n$-GaAs (Minks et al 1989) and of ferrocene at $p$-GaAs (Rosenwaks et al 1992). In the latter case (reduction of ferrocene), the rate constant has been determined by fluorescence decay measurements. Also these large rate constants can only be interpreted assuming adsorption of the electron acceptor as also assumed by Nozik (1993).

So far we have discussed only the determination of rate constants for an electron transfer from the conduction band of an $n$-type semiconductor to an electron acceptor in the dark. As illustrated in figure 6, an anodic photocurrent occurs under illumination. This current is due to a hole transfer from the valence band to an electron donor or due to anodic decomposition. In this case, the anodic current is given by

with

$$
j_{v}^{+}=e k_{v, o x} C_{r e d} p_{s}
$$

$$
p_{s}=p_{L} \exp \left(e \phi_{s c} / k T\right)
$$

where $p_{L}$ is the hole density at the inner edge of the space charge. Since holes are minority carriers in the $n$-type electrode, $p_{L}$ differs from $p_{0}$, and during light excitation $p_{L} \gg p_{0}$. In addition, the photocurrent is entirely determined by the light intensity, 
so that $p_{L}$ or $p_{s}$ are not known. In order to evaluate a rate constant, the anodic process has to be measured with a $p$-type electrode of the same material, in which holes are majority carriers. Since the position of energy bands is usually identical for $n$ - and $p$-type electrodes, the same rate constant should be valid for the $n$-type electrode. A quantitative evaluation is possible by using the quasi-Fermi level concept, as described by Reineke and Memming (1992).

Finally it should be mentioned that the slope of $60 \mathrm{mV} /$ decade of a current-potential curve, as required according to (5), is also obtained when the reaction is diffusioncontrolled (reversible reaction). In this case, the origin of this slope is due to the Nernst law with respect to concentrations of the components of the redox system at the surface (Reineke and Memming 1992). Accordingly, the current-voltage behavior has to be measured and analyzed by using a rotating disc electrode, in order to distinguish between kinetic and diffusion-controlled reactions (Meissner and Memming 1992).

\section{Conclusions}

In the present paper it is shown that, in principle, the same reactions occur at particles and at extended electrodes, but different factors, such as absorption of light, particle size effect, adsorption of the electron donor or -acceptor and formation of a space charge influence the reaction rate. The best way of determining the rate constant is by the analysis of current-voltage curves.

\section{References}

Bahnemann D W 1991 In Photochemical conversion and storage of solar energy (eds) E Pelizzetti and M Schiavello (Amsterdam: Kluwer) p. 251

Bahnemann D W, Henglein A and Spanhel L 1984 Faraday Discuss. Chem. Soc. 78151

Baral S, Fojtik A, Weller H and Henglein A 1986 J. Am. Chem. Soc. 108375

Gerischer H 1960 Z. Phys. Chem. N.F. 26 223, 325

Gerischer H 1961 Z. Phys. Chem. 2748

Gerischer H 1970 In Physical chemistry (eds) M Eyring, W Jost and D Henderson (New York: Academic Press) p. 463, vol. 4A

Gerischer H 1979 In Topics in applied physics (ed.) B O Seraphin (Berlin: Springer Verlag) p. 115

Gomes W P and Cardon F 1982 Prog. Surf. Sci. 12155

Grätzel M and Frank A I 1982 J. Phys. Chem. 862964

Henglein A 1988 Top. Curr. Chem. 143113

Henglein A 1989 Chem. Rev. 891861

Henglein A, Gutiėrrez M and Fischer C H 1984 Ber. Bunsenges. Phys. Chem. 88170

Hodes G 1985 In Energy resources through photochemistry and catalysis (ed.) M Grätzel (New York: Academic Press) p. 521

Jaegermann W and Tributsch H 1988 Prog. Surf. Sci. 291

Lewis N S 1984 Annu. Rev. Mater. Sci. 1495

Lewis N S 1991 Annu. Rev. Phys. Chem. 42543

Lilie I, Beck G and Henglein A 1971 Ber. Bunsenges. Phys. Chem. 75458

Marcus R A 1964 Annu. Rev. Phys. Chem. 15155

Meissner D and Memming R 1992 Electrochim. Acta 37799

Memming R 1983 In Comprehensive treatise of electrochemistry (eds) B E Conway, I O M Bockris and E Yeager (New York: Plenum) vol. 7, p. 529

Memming R 1987 Ber. Bunsenges. Phys. Chem. 91353 
Memming R 1988 Top. Curr. Chem. 14379

Memming R 1990 In Photochemistry and photophysics (ed.) I F Rabek (Boca Raton, FL: CRC Press) vol. 2 , p. 143

Memming R 1991 In Photochemical conversion and storage of solar energy, (eds) E Pelizzetti and M Schiavello (Amsterdam: Kluwer) p. 193

Memming R 1993 Top. Curr. Chem. (in press)

Micic O I, Rajh T and Comor M V 1992 In Electrochemistry in colloids and dispersions (eds) R A Mackay and J Texter (New York: VCH) p. 457

Minks B P, Oskam G, Vanmaekelbergh D and Kelly J J 1989 J. Electroanal. Chem. 273119

Morrison S R 1969 Surf. Sci. 15363

Morrison S R 1980 Electrochemistry at semiconductor and oxidized metal electrodes (New York: Plenum)

Müller B, Majoni S and Meissner D 1993 (MS in preparation)

Nedeljkovic I M, Nenadovic M T, Micic O I and Nozik A I 1986 J. Phys. Chem. 9012

Nozik A I 1993 2nd Int. Conf. Solar Energy Storage and Appl. Photochemistry, Cairo

Pleskov Yu V and Gurevick Y U 1986 Semiconductor photoelectrochemistry (New York: Consultant Bureau)

Reineke R and Memming R 1992 J. Phys. Chem. 96 1310, 1317

Ribarsky M W 1985 In Handbook of optical constants of solids (New York: Academic Press) p. 795

Rosenwaks Y, Thacker B R, Ahrenkiel R K and Nozik A J 1992 J. Phys. Chem. 9610096

Rosetti R, Hall R, Gibson J M and Brus L E 1985 J. Chem. Phys. 82552

Schröder K and Memming R 1985 Ber. Bunsenges. Phys. Chem. 89385

Sze S 1981 Physics of semiconductor devices 2nd edn (New York: John Wiley \& Sons)

Tributsch H 1982 In Solar energy materials. Structure and Bonding Series. Vol. 49. (eds) M J Clarke et al (Berlin: Springer Verlag) p. 127

Tributsch H 1988 In New trends and applications of photocatalysis and photochemistry for environmental problems (ed.) M Schiavello (Dordrecht: D Reidel) p. 297

Uhlendorf I $1993 \mathrm{Ph}$ D thesis, Hamburg

Weller H, Koch U, Gutiérrez M and Henglein A 1984 Ber. Bunsenges. Phys. Chem. 88694 